\title{
Control of galactic scale star formation by gravitational instability or midplane pressure?
}

\author{
Mordecai-Mark Mac Low ${ }^{1}$ \\ ${ }^{1}$ Department of Astrophysics, American Museum of Natural History, Central Park West at \\ 79th Street, New York, NY, 10024-5192, USA. email: mordecai@amnh.org
}

\begin{abstract}
.
Star formation in galaxies has been suggested to depend on large-scale gravitational instability or on the pressure required to form molecular hydrogen. I present numerical models and analysis of observations in support of the gravitational instability hypothesis. I also consider whether the correlation between the surface densities of molecular hydrogen and star formation implies causation, and if so in which direction.
\end{abstract}

Keywords. stars: formation, galaxies: evolution, ISM: molecules, ISM: clouds, ISM: structure

\section{Context}

The importance of understanding galactic-scale star formation has steadily increased as it has become a major stumbling block to confirming the fundamental picture of galaxy evolution in a universe dominated by dark matter and dark energy. The observational study of the question has progressed from the measurements integrated over galaxies of Kennicutt $(1989,1998)$ to the radial profiles of Martin \& Kennicutt (2001) and the subkiloparsec scale pixels of Bigiel et al. (2008). These works have found strong correlations between the surface density of either molecular gas $\Sigma_{\mathrm{H}_{2}}$ or total gas $\Sigma_{\text {gas }}$ and that of star formation $\Sigma_{\mathrm{SFR}}$. The limits of these averaged approaches have been found, however, with the work by Evans (see this volume) showing that individual star forming molecular clouds have star formation rates well above the values derived from integrating over larger scales.

Attempts to predict the star formation rate can broadly be described as taking either global or local approaches to the question, depending on what they propose to be the rate-limiting step in the star formation process. For example, a major class of local models relies on the formation and destruction of molecular clouds as the balance between $\mathrm{H}_{2}$ formation on grains and $\mathrm{H}_{2}$ dissociation by far UV shifts (McKee 1989; Krumholz \& McKee 2005; Shu et al. 2007; Krumholz et al. 2009). Two global models that I will focus on here are global gravitational instability (Martin \& Kennicutt 2001; Rafikov 2001; Kravtsov 2003; Li et al. 2005, 2006; Tasker \& Bryan 2006), and molecular cloud formation determined by the midplane pressure of galactic disks (Elmegreen 1989; Wong \& Blitz 2002; Blitz \& Rosolowsky 2006)

Although proposals for global and local models often present them as opposed to each other, careful examination of the models at each scale reveals that they generally rely on parameters predicted by their counterparts at the other scale, usually through the assumption of empirically determined parameters. For example, global models assume the observed velocity dispersion of the gas and the local star formation efficiency of 
molecular clouds, while local models assume the distribution of cloud properties such as masses, sizes, and locations.

\section{Global Models}

\subsection{Midplane Pressure}

After evaluation of a large set of coordinated observations using The H I Nearby Galaxies Survey (Walter et al. 2008), the Spitzer Infrared Nearby Galaxies Survey (Kennicutt et al. 2003) and the HERACLES CO survey (Leroy et al. 2009), Leroy et al. (2008) concluded that the best predictor of star formation was disk midplane pressure. They relied on two observations. The first, by Wong \& Blitz (2002); Blitz \& Rosolowsky (2004) and Blitz \& Rosolowsky (2006) is that the molecular fraction $R_{\mathrm{mol}}=\Sigma_{\mathrm{H}_{2}} / \Sigma_{\mathrm{H} \text { I }}$ correlates near linearly with the midplane pressure (Elmegreen 1989; Elmegreen \& Parravano 1994)

$$
P_{h} \simeq \frac{\pi}{2} G \Sigma_{\text {tot }}\left(\Sigma_{\text {tot }}+\frac{\sigma_{\text {gas }}}{\sigma_{*, z}} \Sigma_{*}\right) .
$$

where $\sigma_{*}, z$ is the stellar velocity dispersion in the vertical direction, and $\Sigma_{*}$ is the surface density of the stellar disk. The second is that $\Sigma_{\mathrm{SFR}} \propto \Sigma_{\mathrm{H}_{2}}$ (Rownd \& Young 1999; Wong \& Blitz 2002; Gao \& Solomon 2004). The star formation rate can thus be re-expressed as a function of total gas as

$$
\Sigma_{\mathrm{SFR}}\left(\Sigma_{\text {tot }}\right)=\Sigma_{\mathrm{SFR}}\left(\Sigma_{\mathrm{H}_{2}}\right) \frac{R_{\mathrm{mol}}}{R_{\mathrm{mol}}+1} .
$$

The molecular fraction was evaluated by Elmegreen (1993) as being dependent on $P_{h}$ and the FUV radiation field $j$ as $R_{\text {mol }} \propto P_{h}^{2.2} j^{-1}$. If $j \propto \Sigma_{\mathrm{SFR}} \propto \Sigma_{\mathrm{H}_{2}}$, then $R_{\mathrm{mol}} \propto P_{h}^{1.2}$, reproducing the observed correlation.

This raises the question though, of why molecular hydrogen formation should be so important to star formation. The observations of the correlation appear to be on solid ground, at least for galaxies within an order of magnitude or so of the Milky Way in mass. However, the question can be raised of whether correlation implies causation? If so, in which direction does the causation run? Rather than $\mathrm{H}_{2}$ formation being the gate to star formation, perhaps large scale gravitational collapse leads to both $\mathrm{H}_{2}$ formation, and, not much later, to star formation.

Indeed, simulations of $\mathrm{H}_{2}$ formation in a self-gravitating, magnetized, periodic, turbulent box show that (Glover \& Mac Low 2007, 2010) the density enhancements produced by supersonic turbulence can lead to substantial molecular fractions within a few million years. The fraction of molecular hydrogen in these simulations shows a clear dependence on the density of the gas in the box. At a roughly constant temperature (such as the $60 \mathrm{~K}$ characteristic of the cold neutral medium), this also would yield a dependence on pressure.

The evaluation of the star formation law by Leroy et al. (2008) relied on the assumption that the stellar radial velocity dispersion $\sigma_{*, r}$ declined exponentially with radius, based on the apparent constant scale height of stellar disks, and thus exponential decline of $\sigma_{*, z}$. If a constant value of $\sigma_{*, r}$ is instead assumed, however, they find that the next model that I will discuss, gravitational instability, is equally predictive.

\subsection{Gravitational Instability}

Global disk instability models postulate that star formation happens wherever gravitational instability (Gammie 1992; Rafikov 2001) of the combined collisionless stars 
(Toomre 1964) and collisional gas (Goldreich \& Lynden-Bell 1965) in the disk sets in. The criteria for linear instability leading to radial collapse of axisymmetric rings for gas and stellar disks are

$$
Q_{g} \equiv \frac{\kappa c_{g}}{\pi G \Sigma_{\mathrm{tot}}}<1, \quad Q_{*} \equiv \frac{\kappa \sigma_{*, r}}{\pi G \Sigma_{*}}<1.07
$$

where $\kappa$ is the epicyclic frequency, $c_{g}$ the speed of sound, $\sigma_{*}$ the radial stellar velocity dispersion, and $\Sigma_{*}$ is the stellar surface density. (Note that following Rafikov (2001) we use a factor of $\pi$ in the definition of $Q_{*}$ rather than 3.36, shifting the instability criterion to slightly higher value.) Define the dimensionless quantities $q=k \sigma_{*} / \kappa$ and $s=c_{g} / \sigma_{*}$. Then the instability criterion for the combined disk of gas and stars is given by

$$
\frac{2}{Q_{*}} \frac{1}{q}\left[1-e^{-q^{2}} I_{0}\left(q^{2}\right)\right]+\frac{2}{Q_{g}} q \frac{s}{1+q^{2} s^{2}}>\frac{1}{Q_{s g}},
$$

where $I_{0}$ is the Bessel function of order 0 .

\section{Simulations}

Numerical experiments on the behavior of the gravitational instability in disks have been done by $\mathrm{Li}$ et al. (2005), using isothermal gas, collisionless stars, and live dark matter halos computed with GADGET (Springel et al. 2001). They controlled the initial gravitational instability of the disk, and then computed its subsequent behavior. Using sink particles, they measured the amount of gas that collapsed as a function of time, and related it to the minimum radial value of the initial instability $Q_{s g \text {,min }}$. Care was taken to resolve the local Jeans length at all densities below the sink particle threshold to avoid artificial fragmentation (Truelove et al. 1997; Bate \& Burkert 1997), requiring as many as five million particles in their largest simulations.

Kravtsov (2003) and Li et al. (2006) demonstrate that the Schmidt law (Kennicutt 1998 ) is a natural consequence of a gravitationally unstable galactic disk. Kravtsov (2003) computed a cosmological volume and followed the star formation in individual disks, using a star formation law $\dot{M}_{*} \propto \rho_{g}$ deliberately chosen to not automatically reproduce the Schmidt law, as compared to the frequently chosen $\dot{M}_{*} \propto \rho_{g}^{1.5}$. The sink particles used by Li et al. (2006) effectively give a similar star formation law, as they measure collapsed gas above a fixed threshold.

The threshold for sink particle formation corresponds to a pressure $P / k>10^{7} \mathrm{~cm}^{-3}$, corresponding to densities high enough for molecule formation to proceed in under a megayear (Glover \& Mac Low 2007). If the sink particles are interpreted as consisting of mostly molecular gas (the fraction chosen was 70\%), while the SPH particles are treated as atomic gas, the characteristic radial profiles of molecular and atomic gas fond by Wong \& Blitz (2002) are recovered, as is the relation $\Sigma_{\mathrm{SFR}} \propto \Sigma_{\mathrm{H}_{2}}$.

Both Kravtsov (2003) and Li et al. (2006) demonstrated the dropoff from the KennicuttSchmidt Law found by Bigiel et al. (2008) at $\Sigma_{\text {tot }}<10 \mathrm{M}_{\odot} \mathrm{pc}^{-2}$. This reproduction of a major observational result must be counted as a success for the gravitational instability model.

\section{Observational Comparisons}

The first local stability analysis of an external galaxy with resolution below 50 pc was performed by Yang et al. (2007). To measure $\Sigma_{\mathrm{H} \text { I }}$ and $\Sigma_{\mathrm{H}_{2}}$ they used a combined data set of the Australia Telescope Compact Array and the Parkes multibeam receiver (Kim 
et al. 2003) for the H I, and the NANTEN CO survey of the LMC done by Fukui et al. (2001). The rotation curve used to derive $\kappa$ is a fit to the H I and the carbon star (Kunkel et al. 1997) measurements inside and outside of about $3.2 \mathrm{kpc}$, respectively (Kim et al. 1998). To estimate the stellar surface density $\Sigma_{s}$, they used the number density of red giant branch (RGB) and asymptotic giant branch (AGB) stars, follow a procedure similar to that outlined by van der Marel (2001) but use only the Two Micron All Sky Survey Point Source Catalogue (Skrutskie et al. 2006) and different color criteria. They assumed constant values of both $\sigma_{g}$ and $\sigma_{*, r}$, the latter after considering the velocity dispersions of carbon stars, red supergiants, and young globular clusters.

Considering the gas alone, Yang et al. (2007) found that $38 \%$ of the YSOs identified by Gruendl \& Chu (2009) from the Spitzer LMC survey lay in Toomre stable regions. However, when the stellar contribution was included, only $15 \%$ of the YSOs were in stable regions, showing that the stability analysis using the combination of gas and stars provides a reliable way of predicting star formation.

The apparent stability of the M33 disk was raised by Padoan in his talk at this conference as a counterargument to the theory of gravitational instability, with a citation to Corbelli (2003). Examination of that paper shows that the star forming disk of M33 is indeed stable if only the gas is considered. However, Corbelli (2003) also performed a simplified analysis including the stellar surface density, and concluded that the entire star-forming disk is indeed Toomre unstable if the stellar contribution is accounted for (see her Fig. 8).

\section{Conclusions}

Why does midplane pressure appear to work so well at predicting the star formation efficiency of galaxies? The key driver appears to be the linear correlation between $\Sigma_{\mathrm{H}_{2}}$ and $\Sigma_{\text {SFR }}$. But why should $\mathrm{H}_{2}$ exert such a strong influence on star formation? Although $\mathrm{H}_{2}$ is a coolant, interstellar gas can already cool down to below $60 \mathrm{~K}$ with atomic fine structure lines. The remaining cooling is probably not a hugely limiting factor in star formation.

However, gravitational instability produces dense gas that quickly forms $\mathrm{H}_{2}$ (Glover \& Mac Low 2007). Thus, $\mathrm{H}_{2}$, and other molecules such as CO that form with it, may primarily just act to trace dense gas that is already gravitationally unstable and collapsing. Ostriker (this volume) offers an analytic model for how the combination of dynamical and thermodynamical equilibrium may lead to this situation.

Galaxies clearly form stars at widely varying efficiency. Including that in cosmological models seems to be one way forward to understanding the evolution of galaxies over cosmic time (Kravtsov 2010). Varying global efficiency of star formation is a natural consequence of gravitational instability in galaxies with varying properties. The galaxies modeled by Li et al. (2006) show orders of magnitude variation in global efficiency, even with the assumption of constant local efficiency of star formation in any individual collapsing region (an assumption that must ultimately be demonstrated with something looking a lot like a local model, to be sure.)

\section{Acknowledgements}

I thank the organizers for their invitation to speak and partial support of my attendance, and Paolo Padoan for provoking me to check the stability of M33. This work was partly funded by NASA/SAO grant TM0-11008X, by NASA/STScI grant HST-AR11780.02-A, and by NSF grant AST08-06558. 


\section{References}

Bate, M. R. \& Burkert, A. 1997, MNRAS, 288, 1060

Bigiel, F., Leroy, A., Walter, F., Brinks, E., de Blok, W. J. G., Madore, B., \& Thornley, M. D. 2008, AJ, 136, 2846

Blitz, L. \& Rosolowsky, E. 2004, ApJ (Letters), 612, L29

Blitz, L. \& Rosolowsky, E. 2006, ApJ, 650, 933

Corbelli, E. 2003, MNRAS, 342, 199

Elmegreen, B. G. 1989, ApJ, 338, 178

Elmegreen, B. G. 1993, ApJ, 411, 170

Elmegreen, B. G. \& Parravano, A. 1994, ApJ (Letters), 435, L121

Fukui, Y., Mizuno, N., Yamaguchi, R., Mizuno, A., \& Onishi, T. 2001, PASJ, 53, L41

Gammie, C. F. 1992, Ph.D. Thesis, Princeton University, Princeton, NJ, USA

Gao, Y. \& Solomon, P. M. 2004, ApJ, 606, 271

Glover, S. C. O. \& Mac Low, M.-M. 2007, ApJ, 659, 1317

Glover, S. C. O. \& Mac Low, M.-M. 2010, MNRAS, submitted (arXiv:1003.1340)

Goldreich, P. \& Lynden-Bell, D. 1965, MNRAS, 130, 97

Gruendl, R. A. \& Chu, Y.-H. 2009, ApJ Suppl., 184, 172

Kennicutt, R. C. 1989, ApJ, 344, 685

Kennicutt, R. C. 1998, ARAA, 36, 189

Kennicutt, R. C., Jr. et al. 2003, PASP, 115, 928

Kim, S., Staveley-Smith, L., Dopita, M. A., Freeman, K. C., Sault, R. J., Kesteven, M. J., \& McConnell, D. 1998, ApJ, 503, 674

Kim, S., Staveley-Smith, L., Dopita, M. A., Sault, R. J., Freeman, K. C., Lee, Y., \& Chu, Y.-H. 2003, ApJ Suppl., 148, 473

Kravtsov, A. V. 2003, ApJ (Letters), 590, L1

Kravtsov, A. 2010, Adv. Astron., 2010, 1

Krumholz, M. R. \& McKee, C. F. 2005, ApJ, 630, 250

Krumholz, M. R., McKee, C. F., \& Tumlinson, J. 2009, ApJ, 699, 850

Kunkel, W. E., Demers, S., Irwin, M. J., \& Albert, L. 1997, ApJ (Letters), 488, L129

Leroy, A. K., Walter, F., Brinks, E., Bigiel, F., de Blok, W. J. G., Madore, B., \& Thornley, M. D. 2008, $A J, 136,2782$

Leroy, A. K. et al. 2009, AJ, 137, 4670

Li, Y., Mac Low, M.-M., \& Klessen, R. S. 2005, ApJ (Letters), 620, L19

Li, Y., Mac Low, M.-M., \& Klessen, R. S. 2006, ApJ, 639, 879

Martin, C. L. \& Kennicutt, R. C. 2001, ApJ, 555, 301

McKee, C. F. 1989, ApJ, 345, 782

Rafikov, R. R. 2001, MNRAS, 323, 445

Rownd, B. K. \& Young, J. S. 1999, AJ, 118, 670

Shu, F. H., Allen, R. J., Lizano, S., \& Galli, D. 2007, ApJ (Letters), 662, L75

Skrutskie, M. F. et al. 2006, AJ, 131, 1163

Tasker, E. J. \& Bryan, G. L. 2006, ApJ, 641, 878

Truelove, J. K., Klein, R. I., McKee, C. F., Holliman, J. H., Howell, L. H., \& Greenough, J. A. 1997, ApJ (Letters), 489, L179

Toomre, A. 1964, ApJ, 139, 1217

Walter, F., Brinks, E., de Blok, W. J. G., Bigiel, F., Kennicutt, R. C., Thornley, M. D., \& Leroy, A. $2008, A J, 136,2563$

Wong, T. \& Blitz, L. 2002, ApJ, 569, 157

van der Marel, R. P. 2001, AJ, 122, 1827

Yang, C.-C., Gruendl, R. A., Chu, Y.-H., Mac Low, M.-M., \& Fukui, Y. 2007, ApJ, 671, 374 


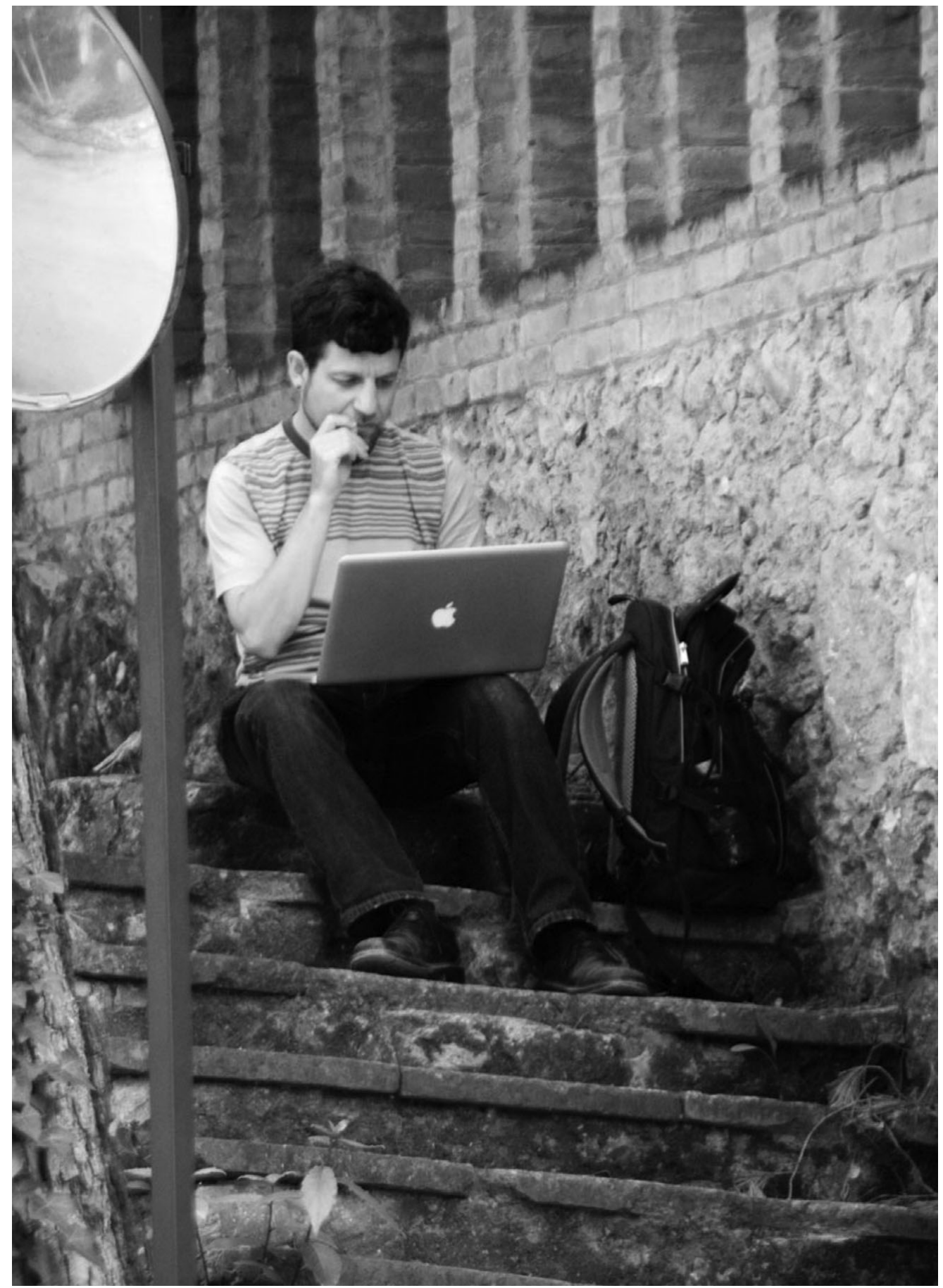

Mordecai Mac Low 